\title{
Androgen up-regulates vascular endothelial growth factor expression in prostate cancer cells via an Sp1 binding site
}

\author{
Kurtis Eisermann ${ }^{1,4}$, Carly J Broderick², Anton Bazarov ${ }^{1}$, Mustafa M Moazam³ and Gail C Fraizer ${ }^{1,2^{*}}$
}

\begin{abstract}
Background: Vascular Endothelial Growth Factor (VEGF) is regulated by a number of different factors, but the mechanism(s) behind androgen-mediated regulation of VEGF in prostate cancer are poorly understood.

Results: Three novel androgen receptor (AR) binding sites were discovered in the VEGF promoter and in vivo binding of AR to these sites was demonstrated by chromatin immunoprecipitation. Mutation of these sites attenuated activation of the VEGF promoter by the androgen analog, R1881 in prostate cancer cells. The transcription factors AR and Sp1 were shown to form a nuclear complex and both bound the VEGF core promoter in chromatin of hormone treated CWR22Rv1 prostate cancer cells. The importance of the Sp1 binding site in hormone mediated activation of VEGF expression was demonstrated by site directed mutagenesis. Mutation of a critical Sp1 binding site (Sp1.4) in the VEGF core promoter region prevented activation by androgen. Similarly, suppression of Sp1 binding by Mithramycin A treatment significantly reduced VEGF expression.
\end{abstract}

Conclusions: Our mechanistic study of androgen mediated induction of VEGF expression in prostate cancer cells revealed for the first time that this induction is mediated through the core promoter region and is dependent upon a critical Sp1 binding site. The importance of Sp1 binding suggests that therapy targeting the AR-Sp1 complex may dampen VEGF induced angiogenesis and, thereby, block prostate cancer progression, helping to maintain the indolent form of prostate cancer.

\section{Background}

In the United States, prostate cancer is the most frequently diagnosed cancer in men with more than 200,000 new cases each year and the second most deadly, killing roughly 30,000 men annually [1]. Prostate cancer growth is dependent upon an adequate blood supply, which is controlled by Vascular Endothelial Growth Factor (VEGF), a regulator of tumor angiogenesis. Several factors are known to modulate VEGF expression including growth factors, cytokines, and hypoxia. Previous studies have also shown that androgen increases VEGF levels [2-5], but the mechanism(s) involved are unknown.

The VEGF promoter lacks a TATA box, is GC rich, and is regulated by multiple transcription factors, such

\footnotetext{
* Correspondence: graizer@kent.edu

'School of Biomedical Sciences, Kent State University, Kent, OH, USA

${ }^{2}$ Department of Biological Sciences, Kent State University, Kent, OH, USA

Full list of author information is available at the end of the article
}

as AP-2, HIF-1, Egr1, and WT1 [6-10]. Previously we have reported the identification of functional WT1 binding sites within the proximal VEGF promoter [7,11], and others have reported interaction of WT1 and HIF1- $\alpha$ in the regulation of VEGF [8]. Additionally, Sp1/Sp3 binding sites located in the core promoter are known to play a role in transcriptional regulation of $V E G F$ in a variety of cell lines including NIH3T3 cells [12], ZR-75 breast cancer cells [13], Y79 retinoblastoma cells [14], NCIH322 bronchioloalveolar cells [15], and PANC-1 pancreatic cells [16]. Members of the Sp family have a conserved C-terminal DNA binding domain, so they can potentially bind the same sequence of DNA and indeed Sp1, 3, and 4 bind preferentially bind at GC-boxes [17]. However, binding at different sites within a promoter region may also confer different functional responses for Sp1 and Sp3 [18]. A cluster of Sp1/3 sites in the proximal promoter mediates regulation of VEGF by TNF- $\alpha$

\section{Biomed Central}


in human glioma cells [19]. Sp1/3 sites are also required for IL-1 $\beta$ induction of VEGF transcription in cardiac myocytes [20] and for TGF- $\beta 1$ stimulation of VEGF transcription in cholangiocellular carcinoma cells [21]. In Panc-1 pancreatic cells, the regulation of VEGF by Sp1 has been extensively documented [16,22] and both constitutive Sp1 activity and a 109 bp core promoter region containing $\mathrm{Sp} 1$ sites are essential for VEGF expression [16]. Overall, the transcriptional regulation of $V E G F$ is cell specific involving different stimuli and factors, but Sp1 plays a prominent role in many cell types.

Since estrogen mediated regulation of VEGF expression in ZR-75 breast cancer cells was shown to require $\mathrm{Sp} 1$ sites in the core VEGF promoter [13], we asked whether androgen might behave similarly in prostate cancer cells. Previous studies have demonstrated that VEGF mRNA levels are elevated by androgen treatment of both human fetal prostatic fibroblasts and LNCaP prostate cancer cells $[2,4,5]$. Also, VEGF protein levels are increased after treatment with hormone [3] and flutamide, an anti-androgen, has been shown to block this up-regulation [23]. However, the hormone responsive region of the VEGF promoter was never identified in these earlier studies, nor was the mechanism of androgen induction of VEGF promoter activity and VEGF mRNA expression determined.

This report characterizing the hormone responsive regions and binding sites within the VEGF promoter is a continuation of earlier studies analyzing conserved putative binding sites in promoters of genes expressed in prostate cancer [11] that identified potentially important non-classical AR binding sites adjacent to other zinc finger transcription factor binding sites in the promoter of $V E G F$ and other genes [24]. Here we identified and characterized the hormone responsive regions of the $V E G F$ promoter, including a required $\mathrm{Sp} 1$ binding site within the core promoter.

\section{Results}

\section{Androgen induces VEGF expression and AR binding to chromatin of prostate cancer cells}

To determine whether VEGF expression was activated by androgen in prostate cancer cells, CWR22Rv1 (22Rv1) cells were treated with the androgen analog R1881. Cells were serum starved overnight and then treated with 5nM R1881 for 48 hours. Figure 1A shows a two-fold increase in VEGF mRNA expression in response to androgen, as measured by quantitative real-time PCR (qRT-PCR). Similar effects were observed in LNCaP cells treated with 1nM R1881 (Additional file 1) and 5nM R1881 (Figure 1B). To confirm that androgen induction of VEGF required hormone-AR interaction, the effect of anti-androgen treatment was then examined using bicalutamide (casodex). LNCaP cells were pre-treated with $0 \mu \mathrm{M}$ or $10 \mu \mathrm{M}$ casodex for $2 \mathrm{hrs}$ and then treated with 5nM R1881 for 24 hours. Casodex treatment significantly reduced the hormone activation of VEGF mRNA indicating that classical signaling requiring ARandrogen interaction was occurring (Figure 1B). Inhibition of hormone induced VEGF expression by casodex was confirmed in 22Rv1 cells (data not shown). Given that hormone enhanced VEGF mRNA levels, VEGF protein expression was also examined in $\mathrm{LNCaP}$ cells treated with androgen. As shown in Figure 1C, VEGF protein expression increased after 1 hour of treatment with 1nM R1881 and maximal expression was seen after 48 hours, which was similar to mRNA expression. Blockade of classical androgen signaling by casodex treatment also decreased hormone mediated up-regulation of cytoplasmic VEGF protein levels by more than $70 \%$. (Figure 1D). To determine whether casodex also blocked a hormone mediated increase in nuclear AR protein levels, nuclear extracts were isolated and western blot analysis was performed. AR protein levels in nuclear lysates prepared from LNCaP cells treated with $0 \mathrm{nM}$ or $1 \mathrm{nM}$ R1881 and with $0 \mu \mathrm{M}$ or $10 \mu \mathrm{M}$ casodex were examined, and casodex was shown to significantly reduce AR protein levels (data not shown).

Having confirmed classical hormone mediated VEGF up-regulation, potential ARE binding sites within the VEGF promoter were then identified using MatInspector software, as previously described [24]. Transcription factor binding site prediction analysis of the VEGF promoter sequence revealed numerous transcription factor binding sites including Sp1, WT1, and Egr1 sites as well as three potential ARE binding sites within $2 \mathrm{~kb}$ of the transcription start site in the VEGF promoter. Since these ARE sites (Figure 2A) were non-classical monomeric sites, it was important that they be tested for functional binding using chromatin immunoprecipitation (ChIP). LNCaP cells were serum starved overnight and then treated with $0 \mathrm{nM}$ or 5nM R1881 for 24 hours. As shown in Figures 2B-D, chromatin of hormone treated LNCaP cells was immunoprecipitated with anti-AR antibody and amplified by three primer sets flanking the regions containing the three putative ARE binding sites (Figure 2A). Hormone treatment enhanced AR binding, as indicated by both standard endpoint PCR (Figures 2B-D) and SYBR Green quantitative qRT-PCR (Figure 2E). Results were quantified as a percentage of input chromatin and showed approximately 2fold increase of chromatin immunoprecipitated by AR antibody in cells treated with $5 \mathrm{nM}$ R1881 compared to that of untreated cells (Figure 2E). These results suggested that all three binding sites were functional and might be important in the hormone regulation of VEGF.

\section{Three non-classical ARE sites contribute to the hormone response of the VEGF promoter}

To determine whether AR binding regions identified by ChIP were transcriptionally activated by hormone, a 

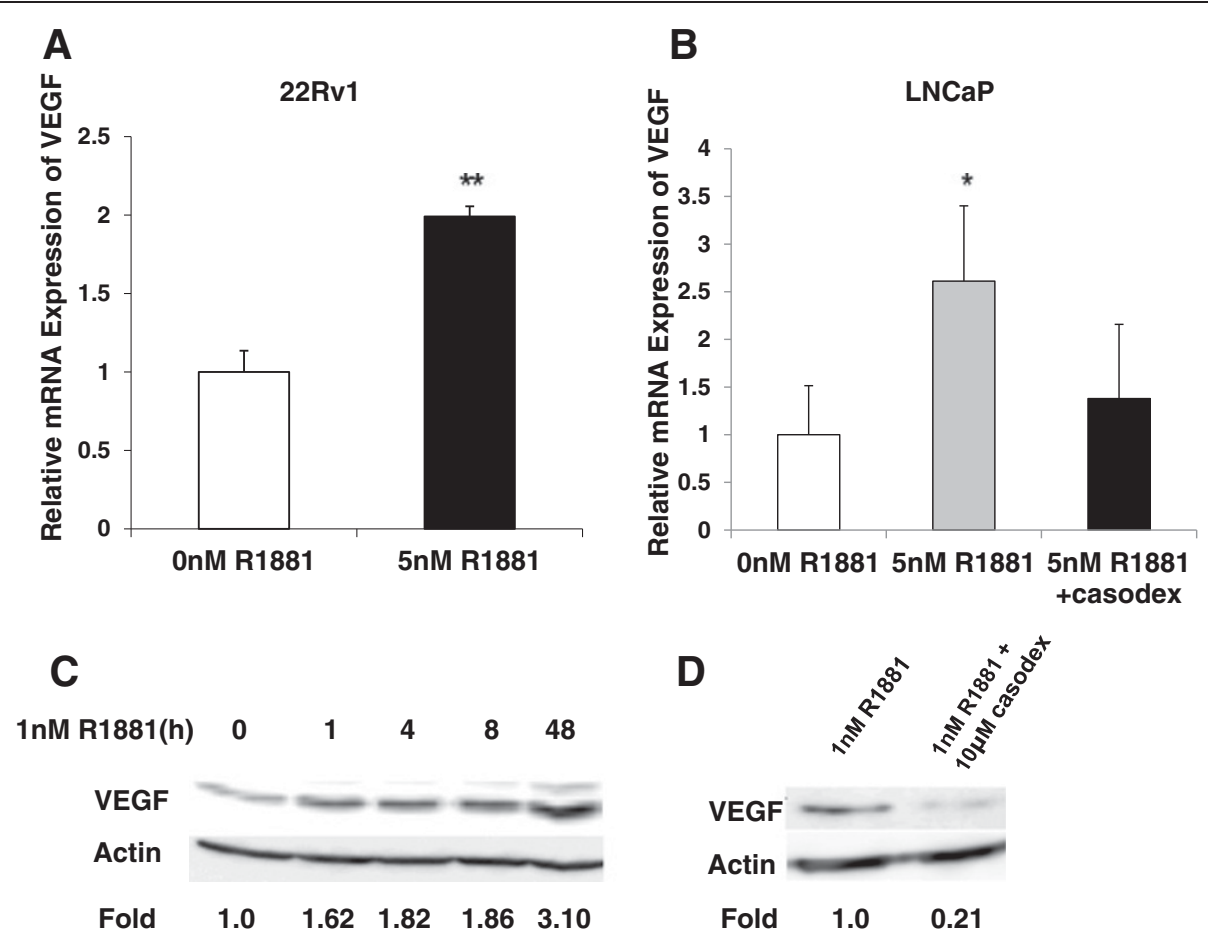

Figure 1 Androgen regulates VEGF expression in LNCaP and 22Rv1 prostate cancer cells. (A) 22Rv1 cells were serum starved overnight then treated with 5nM R1881 or DMSO as a vehicle control (OnM R1881). VEGF mRNA expression was measured by qRT-PCR and normalized by $\beta$-actin levels as described in text. (B) VEGF mRNA expression in LNCaP cells was measured by qRT-PCR and normalized by 185 levels, as described in text. Cells were serum starved as described in A. For inhibition of androgen activity, cells were pre-treated with $10 \mu \mathrm{M}$ casodex for 2 hrs and then induced with 5nM R1881 for 24 hrs. Values represent fold change relative to DMSO treatment. A Student's t-test was performed and significance was determined * $(p<0.05),{ }^{* *}(p<0.01)$. (C) VEGF protein expression in LNCaP cells treated with $1 \mathrm{nM}$ R1881 for 0-48 hours. Protein expression was measured by western blotting as described in text, and $\beta$-actin levels were used as loading controls. (D) Cytoplasmic VEGF protein expression was measured by western blot of LNCaP cells treated as per (B). Image $J$ analysis was performed and VEGF levels were normalized to $\beta$-actin levels. Shown are relative fold-changes in VEGF protein levels, normalized to $\beta$-actin and relative to untreated cells.

series of VEGF promoter deletion constructs were obtained [16] ranging in length from 88 bp (V88) to 2274 bp (V2274). Figure 3A shows the location of predicted Sp1, WT1, Egr1, and AR transcription factor binding sites within the $2 \mathrm{~kb}$ promoter region. These constructs were tested in luciferase assays to determine where within the $V E G F$ promoter the hormone responsive element(s) were located. LNCaP and 22Rv1 cells were transfected with a 411 bp (V411) construct containing only the ARE I site and treated with either increasing doses of R1881 (0.05 to $5 \mathrm{nM}$ ) (Figure $3 \mathrm{~B}$ ) or $5 \mathrm{nM} \mathrm{R} 1881$ with $10 \mu \mathrm{M}$ casodex (Figure 3C and D). After 48 hours, cells were lysed and luciferase assays were performed. 22Rv1 cells were shown to be highly sensitive to androgen as even $0.5 \mathrm{nM}$ R 1881 increased VEGF promoter activity more than 2 fold (Figure 3B). Similarly, in 22Rv1 cells an almost 2 fold increase in VEGF promoter activity was seen in cells treated with 5nM R1881 and casodex blocked this activation (Figure 3C). Additionally, LNCaP cells treated with $5 \mathrm{nM}$ R1881 showed a greater than a 2.5 fold increase in VEGF promoter activity when compared to cells treated with the DMSO vehicle control (Figure 3D). Confirming the requirement for AR-hormone interaction, casodex treatment inhibited this androgen response in LNCaP cells (Figure 3D). Since ARE II and III lie outside of the V411 region, a larger promoter construct (V2274) was examined in $22 \mathrm{Rv} 1$ cells to determine whether hormone activation of VEGF was greater in the $2 \mathrm{~kb}$ reporter construct containing all three ARE binding sites. As shown in Figure 3E, hormone activation of V2274 was increased ( 3.5 fold) in this larger construct, greater than the response shown in the smaller 411 bp reporter construct containing only ARE I. This suggested that all three ARE sites may contribute to androgen activation of the VEGF promoter, although not synergistically.

To determine which ARE sites might be required for androgen mediated up-regulation of the $V E G F$ promoter, all three ARE binding sites were mutated and mutations were confirmed by sequence analysis. Mutations were initially made in the larger V2274 reporter construct which contains all three ARE binding sites (Figure 4A). Site-directed mutagenesis was performed using PCR primers designed to contain base substitutions in either the ARE II or ARE III sites in the V2274 construct (Figure 4B). The effect of 
A

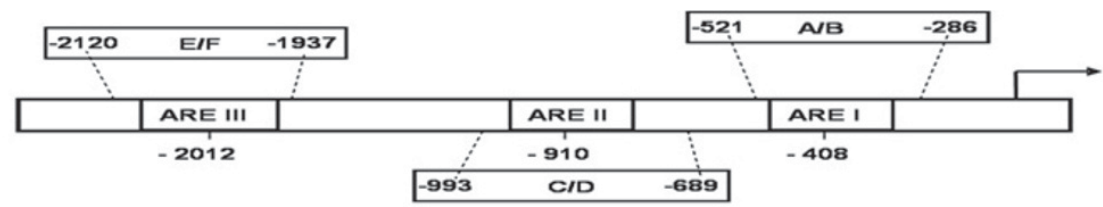

B

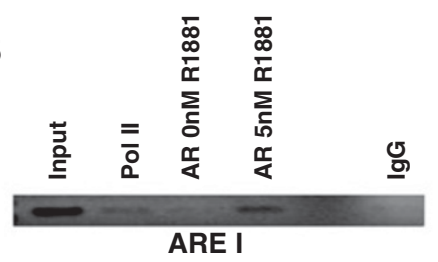

D

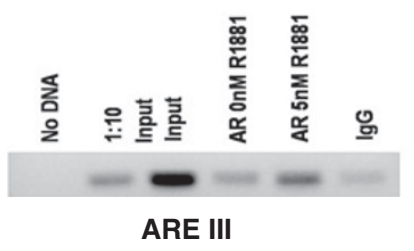

C

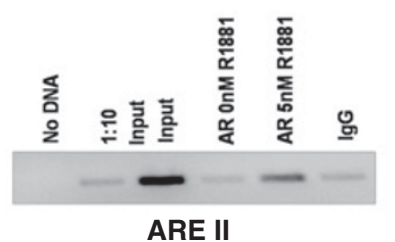

$\mathbf{E}$

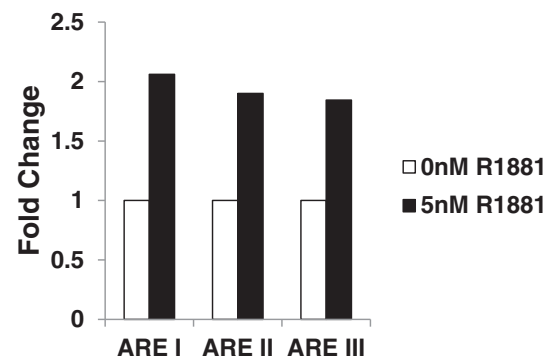

Figure 2 Hormone treatment enhances AR protein binding to the VEGF promoter in chromatin of LNCaP cells. (A) Schematic diagram of the VEGF promoter showing the location of predicted ARE binding sites and primers used to amplify the specific regions of the promoter. (B) ChIP assays were performed with primers specific for the ARE I region of the VEGF promoter using chromatin prepared from LNCaP cells treated for 24 hours with either OnM R1881 or 5nM R1881, following overnight serum starvation. Standard endpoint PCR was performed as described in text. Lane 1 shows amplification of input chromatin that was not immunoprecipitated with antibody, lane 2 chromatin immunoprecipitated with anti-pol II antibody (Upstate), lanes 3 and 4 chromatin from cells treated with OnM or 5nM R1881 and immunoprecipitated with anti-AR (Santa Cruz) antibody and lane 6 is the negative control precipitated with lgG (Upstate). Chromatin amplified in lane 3 was obtained from cells treated with vehicle (DMSO) only. (C) PCR was performed using chromatin as described in (B) and primers specific for the ARE II region (shown in A). Lane 1 is the no DNA control, lane 2 is input diluted 1:10, lane 3 is undiluted input, lanes 4 and 5 chromatin from cells treated with 0nM or $5 n M$ R1881 and immunoprecipitated with anti-AR antibody, and lane 6 is the lgG negative control precipitation. Chromatin amplified in lane 4 was obtained from cells treated with vehicle (DMSO) only. (D) PCR was performed using chromatin as described in (B) and primers specific for the ARE III region. Lanes are the same as in (C). (E) Quantification of immunoprecipitation was performed by SYBR Green qRT-PCR using primers described in (A). Chromatin was immunoprecipitated with anti-AR antibody from LNCaP cells treated with OnM or 5nM R1881 as described above. Average Ct values of immunoprecipitated chromatin were normalized to input and normalized values from $5 \mathrm{nM}$ R1881 treated cells are shown relative to untreated cells (OnM R1881).

eliminating ARE binding at the ARE II or III sites was tested by luciferase reporter assays performed in 22Rv1 cells. Transfections of $22 \mathrm{Rv} 1$ cells were followed by hormone treatment and the wild type V2274 promoter construct was up-regulated approximately 3 fold by $5 \mathrm{nM}$ R1881 and this response was attenuated in the mutant constructs to approximately 2 fold activation (Figures $4 \mathrm{C}$ and D). Double mutation of both ARE II and III sites in V2274 showed similar retention of residual hormone activation when compared to wild type (data not shown). To determine the contribution of ARE I, the V411 reporter construct containing only the ARE I site was mutated as described (Figure 4B) and the effect was tested by luciferase reporter assays performed in both 22Rv1 and LNCaP cells. Figure $4 \mathrm{E}$ shows that in $22 \mathrm{Rv} 1$ cells, the wild type V411 promoter was up-regulated more than 3 fold by
5nM R1881 and this response was attenuated in the mutant ARE I V411 construct to less than 2 fold activation. A similar effect was also seen in LNCaP cells (Figure 4F), although in this case the residual hormone response of the mutant ARE I -V411 construct was not significant. Overall the hormone response of the mutant ARE I promoter was reduced approximately 2-fold in both LNCaP and 22Rv1 cells.

Since single or double mutation of the three ARE sites did not completely eliminate hormone response, we reasoned that one possibility was that all three sites were redundant. However, mutation of two of three ARE sites did not reduce hormone activation to any greater extent than one site alone (data not shown). Another possibility was that other TFs were involved in the hormone response. Thus, we examined the involvement of Sp1, another ZFTF known to regulate VEGF transcription in other systems. 

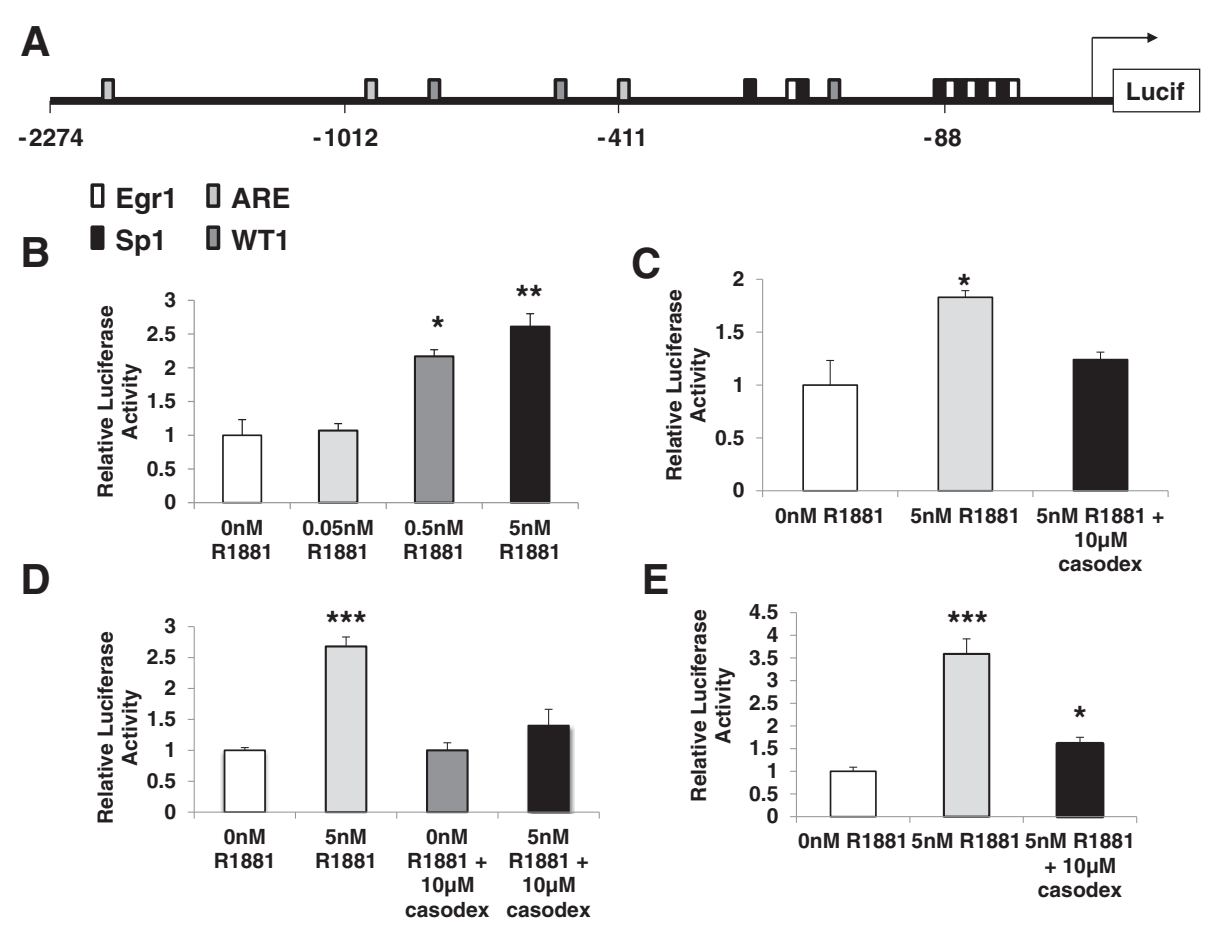

Figure 3 Hormone activates the VEGF promoter in two different cell lines, LNCaP and 22Rv1. (A) Schematic diagram of the VEGF promoter showing locations of predicted ARE binding sites (grey boxes) in relation to 5' termini of luciferase reporter constructs V411 and V2274 [16]. (B) 22Rv1 cells were transfected with the 411 bp VEGF promoter construct (V411) in the presence or absence of different concentrations of R1881 ( 0 , $0.05 \mathrm{nM}, 0.5 \mathrm{nM}$, and $5 \mathrm{nM}$ ) for $48 \mathrm{hrs}$. Luciferase assays of cell lysates were performed and fold activation was determined as described in text. (C)

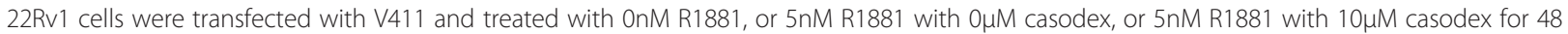
hrs. (D) LNCaP cells were transfected and treated as per (C). (E) 22Rv1 cells were transfected with the 2274 bp VEGF promoter construct (V2274) and treated as per (C). Luciferase activity is shown relative to average normalized luciferase activity in the absence of hormone. Experiments were repeated three times in triplicate. Significance was determined by Student's t-test $\left({ }^{*} p<0.05,{ }^{* *} p<0.01,{ }^{* *} p<0.001\right)$.

\section{Sp1 binding site in the VEGF core promoter is required for hormone responsiveness}

Given that potential Sp1 binding sites were also identified in the VEGF promoter region and Sp1 was observed to bind the VEGF promoter in chromatin of LNCaP cells [11], we asked whether Sp1 binding might contribute to androgen activation of the VEGF promoter. Although the V88 core promoter region is a very G-rich region, containing multiple potential $\mathrm{Sp} 1$ binding sites (Figure $5 \mathrm{~A}$ ), it was not expected to respond to hormone induction, as it does not contain any ARE binding sites. Initially, to test the hormone response of the core promoter, 22Rv1 cells were cotransfected with the V88 reporter construct followed by treatment with R1881. Luciferase assays were performed and surprisingly androgen activated this VEGF core promoter region almost 2 -fold (Figure $5 \mathrm{~B}$ ). To confirm this hormone activation was mediated by AR/androgen interaction, V88 transfected 22Rv1 cells were treated with casodex. Figure $5 \mathrm{C}$ shows that casodex treatment completely blocked the androgen activation of V88, implicating classical androgen signaling at the GC-rich core promoter.

Since the core promoter contains multiple Sp1 binding sites and Mithramycin A is an inhibitor of transcription factor binding to GC rich promoters, we treated 22Rv1 cells with $0.1 \mu \mathrm{M}$ of Mithramycin A for 24 hours to block Sp mediated activation of the VEGF promoter. RNA was isolated and qRT-PCR was performed to determine the effect on VEGF mRNA levels. Figure 6A shows that, as expected, Mithramycin A treatment suppressed VEGF expression as well as $S p 1$, itself. VEGF mRNA levels were repressed more than 5 fold, and $S p 1$ more than 2.5 fold, in the presence of Mithramycin A. To confirm the necessity of $\mathrm{Sp} 1$ binding, site-directed mutagenesis was used to mutate three potential Sp1 binding sites within the core promoter. Primers were designed incorporating mutations for both the Sp1.2 and Sp1.3 binding sites as shown (Figure 6B). Hormone treatment significantly activated the core promoter containing mutant Sp1.2/.3 binding sites ( 2.5 fold) as demonstrated by luciferase assays of R1881 treated 22Rv1 cells (Figure 6B). This suggested that these two potential Sp1 binding sites were not essential for hormone response, so another Sp1 binding site (Sp1.4) closer to the transcriptional start site was examined. Mutation of this site completely suppressed activation by R1881 (Figure 6C). That is, wild-type core promoter was activated by hormone treatment 3 fold, whereas the mSp1.4 

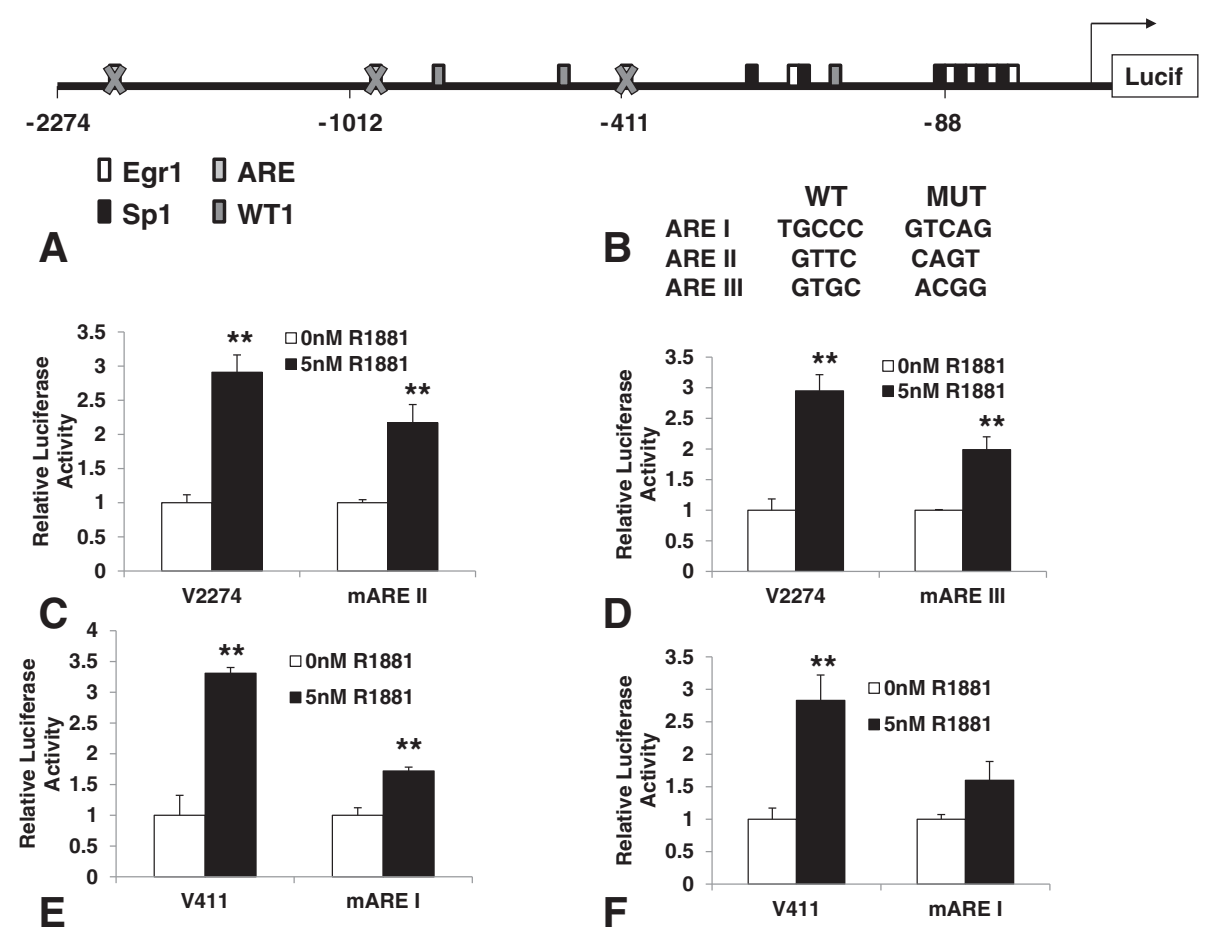

Figure 4 Mutation of ARE sites attenuate hormone activation of the VEGF promoter. (A) Schematic diagram of a $2 \mathrm{~kb}$ region of the VEGF promoter showing the ARE I-III binding sites. Thick X's indicate sites that were mutated. (B) Shown are the wild type (WT) and mutant (MUT) sequences of the ARE I, ARE II, and ARE III binding sites. Site-directed mutagenesis was performed as described in text, using primers containing these mutated bases. (C) Wild type V2274 and mutant (mARE II) luciferase reporters were transfected into 22 Rv1 cells treated with 0nM or 5nM R1881 for 48 hrs. Luciferase assays were performed as previously described. (D) Mutant (mARE III) and wild type V2274 were transfected into 22Rv1 cells as in (C). (E) Wild type and mutant V411 constructs were transfected into 22Rv1 cells as in C. (F) The mutant V411 construct was also transfected into LNCaP cells and treated as in C. Luciferase activity is shown relative to average normalized luciferase activity in the absence of hormone. Experiments were repeated three times in triplicate. Significance was determined by Student's t-test $\left({ }^{* *} p<0.01\right)$.

mutant core promoter was not significantly activated (1.3 fold increase). This reduction of hormone response suggested that the Sp1.4 site was required for a full-strength response of the core promoter to R1881.

To address the possibility that mutation of the Sp1.4 site resulted in a loss of both basal VEGF expression and the induced hormone mediated response we asked whether the mutation altered basal activity. If Sp1 binding at the Sp1.4 site was required for basal transcription, then we would predict both hormone and basal response would be reduced by the mutation. Therefore we compared the basal expression levels of the mutant mSp1.4 promoter construct to the empty vector and found that there was still significant basal transcription occurring (Additional file 2). This data suggests that while the Sp1.4 binding site was essential for hormone response, it was not required for basal activity. Since mutation of Sp1.4 in the V88 construct eliminated androgen induction of the core promoter, it was important that this binding site also be mutated in the V2274 promoter construct to confirm that the $\mathrm{Sp} 1.4$ binding site regulated hormone activation of the full length promoter region, which contains all three ARE binding sites. This was indeed the case, as mutating Sp1.4 within the V2274 construct decreased induction by R1881 (Figure 6D). This was consistent with Figure 6A, which demonstrated transcriptional suppression of the endogenous VEGF promoter by Mithramycin A, an inhibitor of Sp1 binding. Overall the data demonstrated the critical role of the Sp1.4 binding site for hormone mediated activation of VEGF expression.

\section{AR and Sp1 interact in the nucleus and both bind the VEGF core promoter in chromatin}

In addition to these reporter assays showing that the Sp1.4 binding site was required for androgen mediated up-regulation of the $V E G F$ promoter, prior electrophoretic mobility shift assays (EMSA) of the VEGF core promoter had also shown that an oligonucleotide sequence containing the Sp1.4 site bound purified Sp1 proteins in vitro [7]. Here, we determined whether Sp1 would bind the VEGF promoter in vivo. ChIP analysis was performed to determine if both $\mathrm{Sp} 1$ and AR bound to the GC- rich core VEGF promoter in chromatin of hormone treated 22Rv1 cells (Figure 7A). Sp1 and AR immunoprecipitated chromatin was quantified by qRT-PCR and results clearly show that both $\mathrm{Sp} 1$ and AR bound to this 


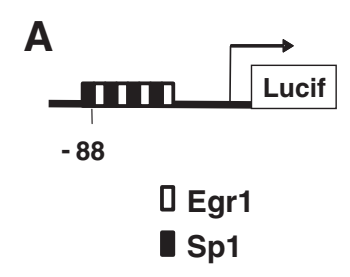

B

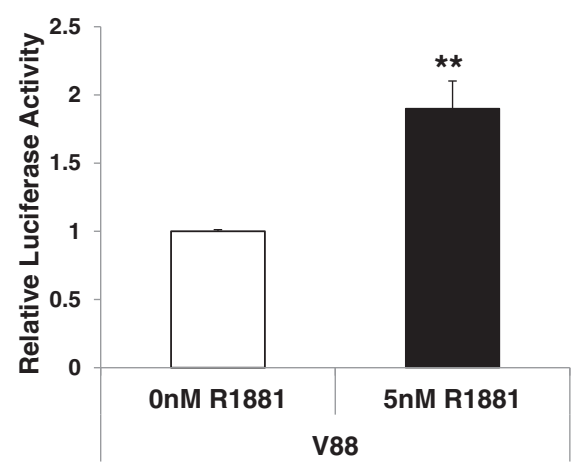

C

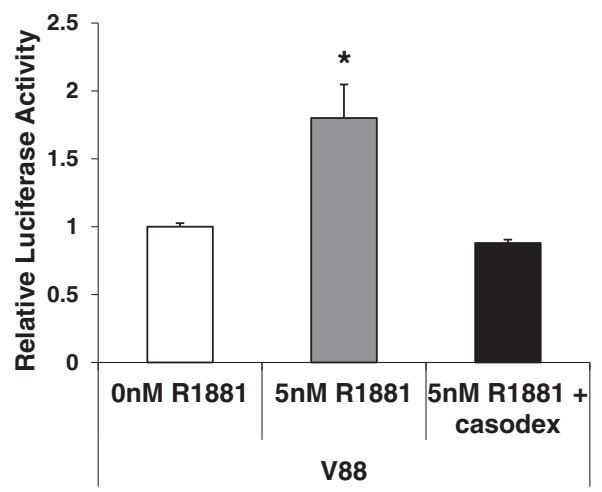

Figure 5 Hormone activates the VEGF minimal core promoter region lacking ARE sites. (A) Schematic diagram of an 88bp region of the VEGF core promoter showing four predicted Sp1 (black box) and Egr1 (white box) binding sites. (B) 22Rv1 cells were transfected with V88 followed by treatment with OnM or 5nM R1881 as described above. Luciferase assays were performed as previously described. (C) 22Rv1 cells were transfected as in (B) except pre-treated with $0 \mu \mathrm{M}$ or $10 \mu \mathrm{M}$ casodex for 2 hours. Luciferase activity is shown relative to average normalized luciferase activity in the absence of hormone. Experiments were repeated three times in triplicate. Significance was determined by Student's t-test $\left({ }^{*} p<0.05,{ }^{* *} p<0.01\right)$.

GC-rich region. In cells treated with 5nM R1881, 10fold more chromatin was immunoprecipitated by Sp1 antibodies than in untreated cells. Similarly, hormone treatment increased AR binding to this region by more than 5 fold, despite the absence of ARE sites. These results indicate that both $\mathrm{AR}$ and $\mathrm{Sp} 1$ were bound to the core VEGF promoter region and suggested the possibility that they might form a complex tethering AR to Sp1 binding sites within the core promoter.

Although AR and Sp1 have previously been demonstrated to interact in LNCaP cells [25], it was unclear whether these two zinc finger transcription factors would form a complex in $22 \mathrm{Rv} 1$ cells. Nuclear lysates from $22 \mathrm{Rv} 1$ cells were immunoprecipitated using AR and Sp1 antibodies as well as a negative control IgG. Using Western blot analysis, the immunoprecipitated proteins were then probed with AR antibody. In the absence of hormone, little AR protein was precipitated by AR antibody and none by Sp1 antibody (Figure 7B, upper panel). In contrast, in the presence of 5nM R1881, AR protein was immunoprecipitated by both $\mathrm{Sp} 1$ and $\mathrm{AR}$ antibodies (Figure 7B, lower panel). This co-immunoprecipitation was hormone specific, occurring only in cells that were treated with $5 \mathrm{nM}$ R1881, indicating that Sp1/AR complex formation was an androgen dependent interaction. This hormone dependent complex formation is consistent with hormone mediated binding and activation of the VEGF core promoter.

\section{Discussion}

We and others have previously demonstrated that androgens up-regulate VEGF expression [3,4,7,23], however, mechanisms involved were not elucidated. Therefore, a molecular understanding of how androgens regulate VEGF in prostate cancer cells was sought. In this study, we firmly established VEGF as a hormone responsive gene and demonstrated that the Sp1.4 binding site located 50 bp downstream of the transcription start site of $V E G F$ was necessary for androgen activation of the VEGF core promoter, a region lacking any potential ARE binding sites, yet responsive to hormone treatment. Consistent with this finding was the observation that Mithramycin A, which inhibits Sp1 binding to GC rich promoter regions, significantly decreased VEGF mRNA levels. Sp1 mediated hormone activation of the VEGF core promoter likely involves both DNA binding and AR protein interaction, as demonstrated by ChIP and co-immunoprecipitation assays. These results support a tethering model for hormone activation of the VEGF core promoter, i.e., that ligand-bound $\mathrm{AR}$ is recruited and then held in place by chromatin-bound Sp1 at the core promoter.

In delineating the hormone responsive regions of the $V E G F$ promoter, we initially focused on three potential ARE sites and demonstrated DNA binding by ChIP and transcriptional activation by R1881. However, mutations of the three functional ARE half-sites merely attenuated, 


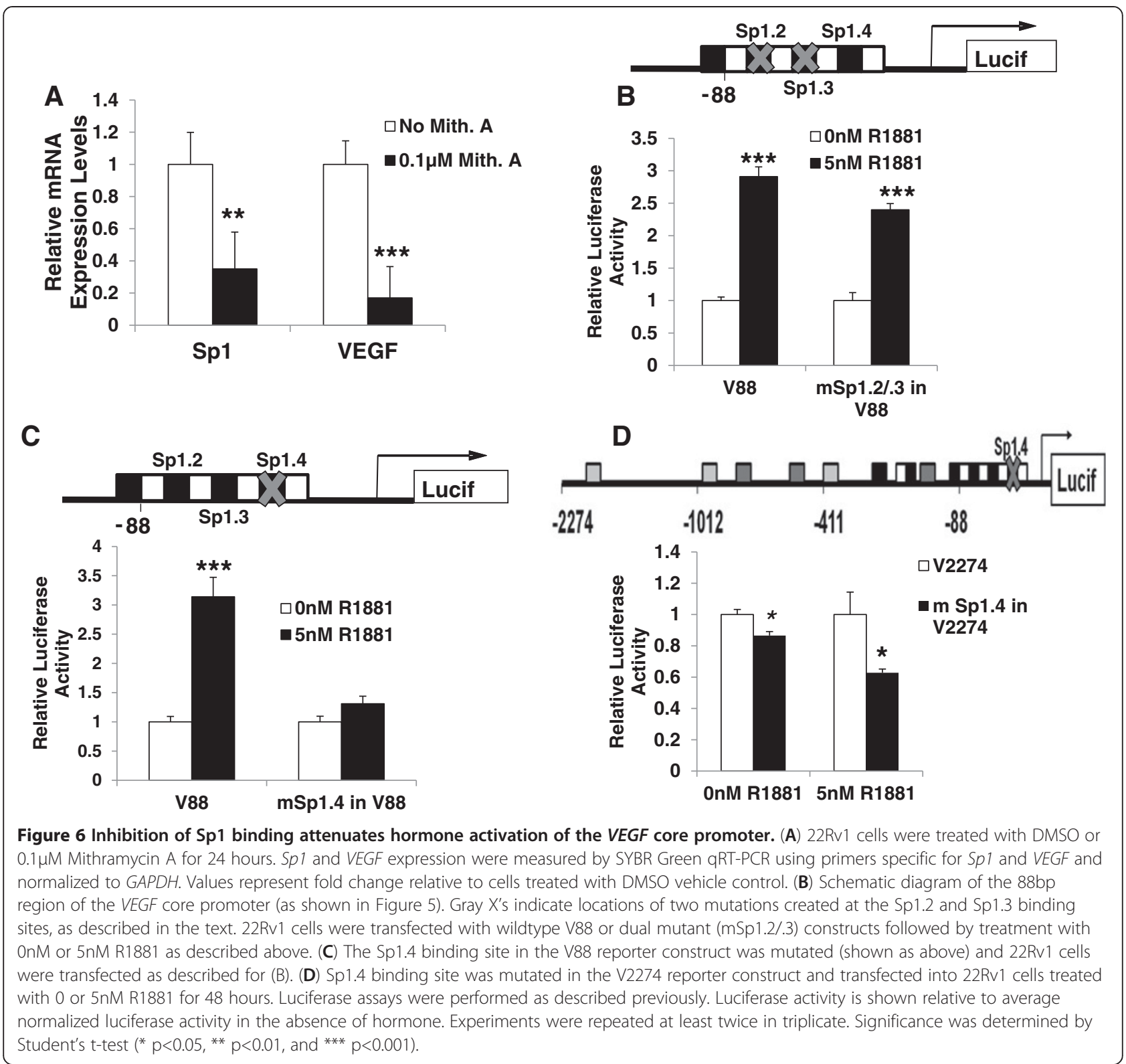

but did not eliminate activation of VEGF by androgen. Androgens are known to regulate a multitude of genes, with the most well studied androgen regulated gene being PSA. There are three known dimeric ARE binding sites in the regulatory region of PSA [26] and, similar to the VEGF promoter, all three sites are involved in androgen regulation. Although mutation of the two AREs in the proximal promoter of PSA significantly decreased activation by R1881, mutation of the ARE in the distal enhancer located $4 \mathrm{~kb}$ upstream of the transcription start site completely blocked androgen activation of PSA. In contrast to PSA, the ARE sites in the VEGF promoter are monomeric sites, not canonical dimeric ARE sequence, and are located within $2 \mathrm{~kb}$ of the start site. One possibility is that binding at these non-classical sites in the VEGF promoter may not be as strong as that in the PSA promoter, but interaction with other TFs might enhance or stabilize binding of AR, increasing VEGF expression.

Since the VEGF promoter region is highly GC-rich we investigated the role of other zinc finger transcription factors known to bind GC-rich promoter regions, such as Sp1. Sp1/Sp3 binding sites in the core promoter region are known to control VEGF transcriptional regulation in a number of different cell lines. Sp1 mediates regulation of VEGF in the presence of specific stimuli, such as stress [27], estrogen [13], retinoic acid [14], TGF- $\beta 1$ [21], and PDGF [12] depending on the cell type. Androgens have been known to act in concert with other zinc finger transcription factors such as GATA [28] and Sp1 [25,29] to regulate androgen responsive genes such as PSA, $p 21$, 


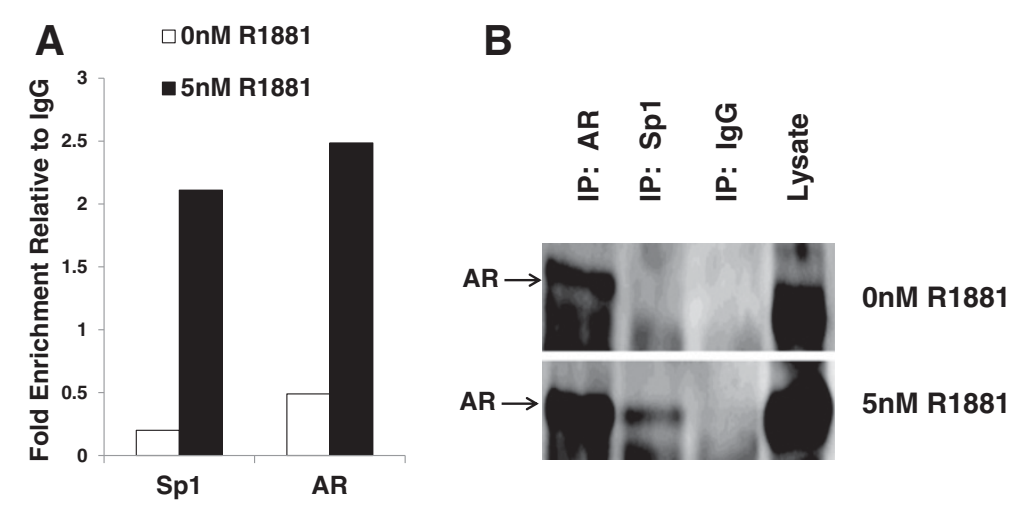

Figure 7 AR and Sp1 form a complex and bind to the VEGF core promoter. (A) Quantitation of AR and Sp1 immunoprecipitated chromatin was performed by qRTPCR amplification of the VEGF core promoter region following ChIP assay of 22Rv1 cells treated with 0nM or 5nM R1881 for 48 hours prior to harvest, as described in Figure 2. Chromatin was immunoprecipitated with AR (Santa Cruz) or Sp1 (Upstate) antibodies, or IgG (Upstate), then quantified by SYBR-Green qRT-PCR using primers specific for the VEGF core promoter region (as described in text). Values shown are fold enrichment of Ab specific immunoprecipitations compared to IgG controls. (B) The interaction of AR and Sp1 in hormone treated 22Rv1 cells was demonstrated by co-immunoprecipitation of cells treated with OnM or 5nM R1881 for 48 hours prior to harvest. Nuclear extracts were incubated with either AR Ab (Santa Cruz), Sp1 Ab (Upstate), or serum IgG and the complexes were collected by sepharose magnetic beads (Active Motif, CA). Proteins eluted from the beads were denatured and electrophoresed, then blots were probed with AR Ab. Top and bottom panels show proteins immunoprecipitated from cells treated with OnM or 5nM R1881, respectively.

and NRIP. Previously, Sp1 sites have been shown to be involved in androgen induction of both the $p 21$ gene and the NRIP (nuclear receptor interaction protein) gene and co-IP demonstrated that AR interacts with $\mathrm{Sp} 1$ to regulate their expression [25,29]. Similarly we have demonstrated that $\mathrm{Sp1}$ plays a role in the androgen responsiveness of $V E G F$ by forming a complex with AR and binding to the $V E G F$ promoter. While there are four Sp1 binding sites in the core promoter region, mutation of a single binding site, Sp1.4, eliminated androgen induction of this region of VEGF. The VEGF promoter is similar to the NRIP promoter, in that both are TATA-less GC rich promoters that are induced by androgen in prostate cancer cells [29]. The NRIP promoter also contains three Sp1 sites and two hormone responsive elements (ARE and GRE). Similar to our findings in the VEGF promoter, mutation of these ARE/ GRE sites did not eliminate hormone response; and Sp1 and AR were shown to cooperatively interact by several methods, including sequential chromatin immunoprecipitation and co-IP. In both these promoters, the association of AR with $\mathrm{Sp} 1$ appeared to cooperatively regulate promoter activity.

The mechanism of androgen mediated regulation of $V E G F$ identified by this study is analogous to estrogen mediated regulation of VEGF in breast cancer cells [13]. We show here that androgen up-regulates the $V E G F$ core promoter, a region lacking ARE binding sites, but containing four binding sites in which Sp1 or Sp3 can bind. In ZR-75 breast cancer cells, estrogen regulation of $V E G F$ expression is thought to act through ER- $\alpha / \mathrm{Sp} 1$ and ER- $\alpha / \mathrm{Sp} 3$ interactions with GC-rich motifs [13]. These authors showed that treatment with estradiol increased VEGF mRNA levels greater than fourfold. Additionally, the GC-rich region of the VEGF core promoter ( -66 to -47 ) was required for E2 activation of $V E G F$, despite a lack of classical ER binding sites. Both Sp1 and Sp3 were demonstrated to bind the VEGF promoter in vitro by EMSA and in vivo by ChIP, further supporting their functional relevance in E2-mediated regulation of VEGF. While these non-classical mechanisms of hormone mediated VEGF regulation operate under normoxic conditions, under hypoxic conditions HIF- $1 \alpha$ is known to regulate VEGF expression. Androgen regulation of VEGF by HIF- $1 \alpha$ is thought to occur indirectly through an autocrine loop involving EGF/ phosphatidylinositol 3 '-kinase/protein kinase $\mathrm{B}$, which activates HIF- $1 \alpha$ and HIF- $1 \alpha$ regulated expression of $V E G F$ under hypoxic conditions [23].

\section{Conclusions}

Androgen mediated regulation of VEGF expression required a specific $S p 1 / 3$ binding site in the $\mathrm{GC}$-rich VEGF core promoter. Although ARE sites within the $V E G F$ promoter bound AR and their mutation dampened VEGF expression, mutation of a key Sp1 binding site in the core promoter of VEGF blocked promoter activation by hormone. Our findings with androgen reflect those of others examining regulation of $V E G F$ by other hormones [13-15]; overall these studies demonstrate the complexity of hormone activation of VEGF and the importance of protein-protein interactions. Regulation of $V E G F$ by zinc finger transcription factors, such as Sp1, and the importance of their interactions with AR, suggests that they may play a positive role in promoting 
angiogenesis and prostate cancer progression. Thus, elevated expression of these zinc finger transcription factors may indicate a worse prognosis. Therapy disrupting ARSp1 complexes and thereby suppressing VEGF would be expected to limit angiogenesis and maintain the indolent form of prostate cancer.

\section{Methods}

Cell culture and hormone treatment

LNCaP (ATCC CRL-1740) and CWR22Rv1 (ATCC CRL-2505) prostate cancer cells were cultured in RPMI media. All cells were grown in media supplemented with $10 \% \mathrm{FCS}$ and $100 \mathrm{ug} / \mathrm{ml}$ penicillin/streptomycin in a $37^{\circ} \mathrm{C}$ incubator with $5 \% \mathrm{CO}_{2}$. For hormone treatment, cells were grown to $60-80 \%$ confluency and then serum starved overnight in either serum-free media or media supplemented with 5\% charcoal-dextran stripped FBS RPMI. The synthetic androgen methyltrienolone (R1881) (Perkin Elmer, Boston, MA) was then added to the charcoaldextran stripped FBS RPMI media and cells were treated with 5nM R1881 for 24 hours unless otherwise noted in figure legends. For inhibition of AR, $10 \mu \mathrm{M}$ bicalutamide/ casodex (LKT Labs, St. Paul, MN) was added 2 hours prior to treatment with R1881.

\section{Chromatin immunoprecipitation}

Two million cells were treated with formaldehyde to crosslink proteins to DNA and lysed as per manufacturer's recommendations using Millipore EZ ChIP Assay (Upstate Biotechnology Inc., Billerica, MA). Chromatin was sheared by sonication (Biosonik III, Bronwill Scientific, Rochester, NY) to fragments of $200-1,000$ bp in length. The supernatant was pre-cleared by incubation with Protein G Agarose and incubated overnight at $4^{\circ} \mathrm{C}$ with either anti- AR (Santa Cruz), Sp1 (Santa Cruz) and control polymerase II antibodies or non-immune IgG (Upstate Biotechnology Inc.). The complexes were recovered from Protein G magnetic beads, crosslinks were reversed and DNA was purified. Four percent of both immunoprecipitated and input chromatin were amplified by PCR using Taq polymerase (Applied Biosystems by Roche Molecular System, Inc) and the appropriate primers (ARE I (FOR): 5'-TTCGAGAGTGAGGACGTGTG-3', ARE I (REV): 5' AGGGAGCA GGAAA GTGAGGT-3', ARE II (FOR): $5^{\prime}$ TCACTGACTAACCCCGGAAC-3', ARE II (REV): $5^{\prime}$-TT TGG GACTGGAGTTGCTTC-3', ARE III (FOR): $5^{\prime}$ GGCTCTTTTAGGGGCTGAAG-3', ARE III (REV): $5^{\prime}$ AGGCTGATGAACGGGATATG-3', VEGF V88 (FOR) 5'CCGCGGGCGCGTGTC TCTGG-3', VEGF V88 (REV) $5^{\prime}$-TGCCCCAAGCCTCCGCGATCCTC-3'). Following an initial 10 min denaturation at $95^{\circ} \mathrm{C}$, DNA was amplified by $32-35$ cycles of: 1$) 20 \mathrm{sec}$ denaturation at $95^{\circ} \mathrm{C}$, 2) 30 sec annealing at either $52^{\circ} \mathrm{C}$ (for ARE III primers), $53^{\circ} \mathrm{C}$ (for ARE II primers), $58^{\circ} \mathrm{C}$ (for ARE I and VEGF V88 primers) and 3) $30 \mathrm{sec}$ extension at $72^{\circ} \mathrm{C}$; amplification was completed with a 2 min final extension at $72^{\circ} \mathrm{C}$. PCR products were electrophoresed on $1 \%$ agarose gel, and ethidium bromide stained DNA was visualized by a gel doc system (Biorad, Hercules, CA).

For quantitation of immunoprecipitated chromatin by quantitative real-time PCR (qRT-PCR), purified DNA samples were amplified in an ABI 7000 thermocycler using primers listed above and following manufacturer's recommendation for SYBR Green Q-PCR (Applied Biosystems, Foster City, CA). Each PCR reaction was carried out in triplicate and average $\mathrm{Ct}$ values were normalized to total input (non- immunoprecipitated) DNA. The amount of DNA immunoprecipitated with the target antibody from hormone treated cells R1881 was compared to that of control samples treated only with vehicle. Shown are input normalized $\mathrm{Ct}$ values from chromatin of treated cells relative to untreated control cells.

\section{RNA isolation and quantitative real-time PCR}

RNA was isolated from subconfluent cells using the GenElute Mammalian Total RNA Miniprep Kit (Sigma, St. Louis, MO) as per manufacturer's recommendation. Following quantitation, $1 \mu \mathrm{g}$ of RNA was reverse transcribed using the High Capacity cDNA ReverseTranscription Kit (Applied Biosystems, Carlsbad, CA). qRT-PCR was performed using either Taqman Universal Master Mix with pre-designed Taqman Gene Expression Assay probe sets for VEGFA (Hs00900057_m1) and 18S (Hs99999901_s1) or SYBR Green Master Mix with primers specific for VEGF, Sp1, GAPDH, and Beta-actin: VEGF (FOR): 5'-CG AAACCATGAACTTTCTGC-3', VEGF (REV): 5'-CCT CAGTGGGCACACACTCC-3', Sp1 (FOR) 5'-TGCATTT CAAGGAATGGAAT-3', Sp1 (REV) 5'-GCTTCCTTGGT GTGAAGAGA-3', GAPDH (FOR): 5'-CCATCACCATC TTCCAGGAG-3', GAPDH (REV): 5'-GGATGATGTTCT GGAGAGCC-3', Beta-actin (FOR): 5'-GTGGGGCGCC CCA GGCACCA-3', Beta-actin (REV): 5'-GTCCTTAAT GTCACGCACGATTTC-3'). The comparative Ct method [30] was used to analyze gene expression differences between control (untreated) cells and cells treated with R1881 alone or with the anti-androgen casodex.

\section{Western blot}

Subconfluent monolayers of LNCaP and 22Rv1 cells were washed in PBS and proteins were extracted using RIPA lysis buffer (1\% NP40, 0.5\% Na Deoxycholate, 0.1\% SDS, and $150 \mathrm{mM} \mathrm{NaCl}$ ) containing protease inhibitors. To quantify the amount of proteins present in each lysate, bicinchoninic acid (BCA) assays (Pierce, Thermo Fisher Scientific, Rockford, IL) were performed and absorbance was measured at $600 \mathrm{~nm}$ on a Dynex Technologies (Chantilly, VA) MRX Revelation plate reader. Proteins (25-50ug) were separated by sodium dodecyl 
sulfate polyacrylamide gel electrophoresis (SDS-PAGE). After transfer to a Polyvinylidene Fluoride (PVDF) membrane and blocking with $5 \%$ casein, blots were probed overnight at $4^{\circ} \mathrm{C}$ with polyclonal VEGF and $\mathrm{AR}$ antibodies (Santa Cruz), and monoclonal $\beta$-actin antibody (GenScript). Washed blots were then incubated for $1 \mathrm{hr}$ in either HRPconjugated anti - rabbit (GenScript) or anti - mouse (Santa Cruz) antibodies. Proteins were visualized by incubating the membrane in a luminol ECL solution followed by chemiluminescent detection using a Fuji LAS 3000 (GE, Piscataway, NJ) detection system. Bands were quantified using ImageJ analysis and normalized to actin levels.

\section{Co-Immunoprecipitation}

Nuclear extracts from 22Rv1 cells were prepared using Active Motif's Universal Magnetic Co-IP kit (Carlsbad, CA) as per manufacturer's recommendations. Cells were swelled in Hypotonic Buffer containing phosphatase-, deacetylase-, and protease- inhibitors, then lysed in 5\% detergent. This suspension was then centrifuged at $14,000 \mathrm{x} g$ and the supernatant was discarded leaving the nuclear fraction which was enzymatically sheared in the presence of the same inhibitors. Nuclear extracts $(150-200 \mu \mathrm{g})$ were then combined with $5 \mu \mathrm{g}$ of either AR (Santa Cruz) or Sp1 (Upstate) antibodies, or negative control IgG in the presence of the same inhibitors. Following antibody incubation, complexes were pulled down with Protein G magnetic beads. After washing, these complexes were separated by SDS-PAGE and identified by Western blot analysis (as described above).

\section{Plasmid Transfection and Luciferase Assay}

The pGL3-VEGF luciferase reporter constructs (V88, V411, or V2274) were generously provided by Dr. Xie [16] and DNA purified by the Qiagen plasmid Maxi prep kit (Qiagen, Valencia, CA). LNCaP and 22Rv1 cells were plated in 12-well plates as described above. After reaching $~ 70-$ 90\% confluency, cells were serum-starved for 18-24 hours with serum-free RPMI and then transfected with VEGF reporter constructs using Lipofectamine 2000 (Invitrogen, Carlsbad, CA) as described [7]. After 4-6 hours, transfection media was replaced with appropriate growth media. For hormone induction, 5nM R1881 was added to media with $5-10 \%$ charcoal-dextran stripped FBS as described above. After 48 hours cells were lysed and luciferase activity was measured using a Promega luciferase assay kit (Promega, Sunnyvale, CA) and a Turner luminometer (Promega, Sunnyvale, CA) following manufacturer's recommendations. The luciferase activity was normalized to total cell protein, using a micro BCA protein assay, as described above. All experiments were done in triplicate and repeated at least three times. Standard errors of the mean were determined using GraphPad InStat software (San Diego, CA). Significance was determined by Student's t-test.

\section{Site directed mutagenesis}

Potential binding sites in the VEGF promoter were identified using MatInspector, as previously described [11]. Predicted AR and Sp1 binding sites in the VEGF promoter construct were then mutated using the QuikChange SiteDirected Mutagenesis Kit (Stratagene, Agilent Technologies, Santa Clara, CA). Primers were designed according to the manufacturer's suggestions using the QuikChange Primer Design Program. Primers containing the desired mutation (shown in bold) are listed below:

(ARE I (FOR):

5'-CTCTATCGATAGGTACCGTGGTCAGCTCTCCC C ACCCGTC CCTGTC-3',

ARE I (REV): 5'GACAGGGACGGGTGGGGAGAGCT GACCACGGTACCTATCGA TAGAG-3',

ARE II (FOR): 5'-GGAACCACACAGCTTCCCACTGT CAGCTCCACA AAC TTGG-3',

ARE II (REV): 5'-CCAAGTTTGTGGAGCTGACAGT GGGAAGCTGTGTGGTTCC-3',

ARE III (FOR): 5'-GCCCCAAGATGTCTACAGCTTA CGGTCCTGGGGTGC-3',

ARE III (REV): 5'-GCA CCCCAGGACCGTAAGCTG TAGACATCTTGGGGC-3',

Sp1.2/Sp1.3 (FOR): 5'-GCCCC CCG GTT CGGGCCG GGTTCGGGGTCCC-3',

Sp1.2/Sp1.3 (REV): 5’-GGGACCCCG AACC CGG CCC GAA CCGGGGGGC-3',

Sp1.4 (FOR): 5'-GGGTCCCGGCGGTTCGGAGCCAT GCG-3',

Sp1.4 (REV): 5'-CGCATGGCTCCG $A$ ACCGCCGGGA CCC-3').

PCR was performed using the V88, the V411, or the V2274 luciferase reporter constructs and the appropriate mutant primers. After PCR amplification, parental strands were digested with DpnI and XL1-Blue super competent cells were transformed with remaining mutant DNA. Individual colonies were grown, plasmids were purified (Qiagen, Valencia, CA) and sequenced to verify that the correct base pairs were changed (Cleveland Clinic Genomics Core, Lerner Research Institute, Cleveland, $\mathrm{OH}$ ). Luciferase assays were performed using mutant constructs as described above.

\section{Additional files}

Additional file 1: Figure S1. R1881 (1nM) induces VEGF mRNA expression in LNCaP cells. LNCaP cells were serum starved overnight followed by treatment with either OnM R1881 (DMSO) or 1nM R1881 for 48 hours. VEGF mRNA expression was measured by qRT-PCR and normalized to $18 \mathrm{~S}$ levels as described. Values represent fold change relative to DMSO treatment. A Student's t-test was performed and significance was determined ${ }^{* *}(p<0.01)$

Additional file 2: Figure S2. Mutation of the Sp1.4 binding site does not eliminate basal activity of the VEGF core promoter. 22Rv1 cells were transfected with mSp1.4 (in V88 core promoter construct) or pGL3- Basic 
empty vector. Cells were transfected and luciferase assays were performed as described. Experiments were performed in triplicate and repeated twice. Luciferase activity is shown relative to average normalized activity of the pGL3-Basic empty vector. Significance was determined by Student's t-test ${ }^{* * *}(p<0.001)$.

\section{Competing interests}

The authors declare that they have no competing interests.

\section{Authors' contributions}

KE and GF conceived and designed study; analyzed and interpreted data; drafted and revised the manuscript. KE performed experiments and the statistical analyses. CJB and MM assisted with luciferase and GRT-PCR assays. $A B$ assisted with sequence analyses and co-immunoprecipitations. All authors read and approved the manuscript

\section{Acknowledgements}

We gratefully acknowledge the generosity of Dr. Keping Xie for providing the pGL3-VEGF luciferase reporter constructs (V88, V411, and V2274). Funding was provided by NIH-1CA33160 (GF), Sigma Xi G2009101485 (KE), Kent State University Graduate Student Senate (KE) and the CHMCA Foundation (MM).

\section{Author details}

'School of Biomedical Sciences, Kent State University, Kent, OH, USA.

${ }^{2}$ Department of Biological Sciences, Kent State University, Kent, OH, USA. ${ }^{3}$ Department of Pediatric Hematology/Oncology, Akron Children's Hospital, Akron, $\mathrm{OH}$, USA. ${ }^{4}$ Department of Urology, University of Pittsburgh, Pittsburgh, PA, USA.

Received: 23 May 2012 Accepted: 21 January 2013 Published: 1 February 2013

\section{References}

1. Jemal A, Siegel $R, X u J$, Ward E: Cancer statistics, 2010. CA: Cancer J Clin 2010, 60(5):277-300.

2. Levine AC, Liu XH, Greenberg PD, Eliashvili M, Schiff JD, Aaronson SA, Holland JF, Kirschenbaum A: Androgens induce the expression of vascular endothelial growth factor in human fetal prostatic fibroblasts. Endocrinology 1998, 139(11):4672-4678.

3. Joseph IB, Nelson JB, Denmeade SR, Isaacs JT: Androgens regulate vascular endothelial growth factor content in normal and malignant prostatic tissue. Clin Cancer Res 1997, 3(12 Pt 1):2507-2511.

4. Li J, Wang E, Rinaldo F, Datta K: Upregulation of VEGF-C by androgen depletion: the involvement of IGF-IR-FOXO pathway. Oncogene 2005, 24(35):5510-5520.

5. Stewart RJ, Panigrahy D, Flynn E, Folkman J: Vascular Endothelial Growth Factor expression and tumor angiogenesis are regulated by androgens in hormone responsive human prostate carcinoma: evidence for androgen dependent destabilization of vascular endothelial growth factor transcripts. J Urol 2001, 165(2):688-693.

6. Ruiz M, Pettaway C, Song R, Stoeltzing O, Ellis L, Bar-Eli M: Activator protein $2 a$ inhibits tumorigenicity and represses vascular endothelial growth factor transcription in prostate cancer cells. Cancer Res 2004, 64(2):631.

7. Hanson J, Gorman J, Reese J, Fraizer G: Regulation of vascular endothelial growth factor, VEGF, gene promoter by the tumor suppressor, WT1. Front Biosci 2007, 12:2279-2290.

8. McCarty G, Awad O, Loeb DM: WT1 protein directly regulates expression of vascular endothelial growth factor and is a mediator of tumor response to hypoxia. J Biol Chem 2011, 286:43634-43643.

9. Lee KH, Kim JR: Hepatocyte growth factor induced up-regulations of VEGF through Egr-1 in hepatocellular carcinoma cells. Clin Exp Metastasis 2009, 26(7):685-692.

10. Forsythe JA, Jiang BH, lyer NV, Agani F, Leung SW, Koos RD, Semenza GL: Activation of vascular endothelial growth factor gene transcription by hypoxia-inducible factor 1. Mol Cell Biol 1996, 16(9):4604-4613.

11. Eisermann K, Tandon S, Bazarov A, Brett A, Fraizer G, Piontkivska H: Evolutionary conservation of zinc finger transcription factor binding sites in promoters of genes co-expressed with WT1 in prostate cancer. BMC Genomics 2008, 9:337.

12. Finkenzeller G, Sparacio A, Technau A, Marme D, Siemeister G: Sp1 recognition sites in the proximal promoter of the human vascular endothelial growth factor gene are essential for platelet-derived growth factor-induced gene expression. Oncogene 1997, 15(6):669-676.

13. Stoner M, Wormke M, Saville B, Samudio I, Qin C, Abdelrahim M, Safe S: Estrogen regulation of vascular endothelial growth factor gene expression in ZR-75 breast cancer cells through interaction of estrogen receptor a and SP proteins. Oncogene 2004, 23:1052-1063.

14. Akiyama H, Tanaka T, Maeno T, Kanai H, Kimura Y, Kishi S, Kurabayashi M: Induction of VEGF gene expression by retinoic acid through Sp1-binding sites in retinoblastoma Y79 cells. Invest Ophthalmol Vis Sci 2002, 43(5):1367.

15. Maeno T, Tanaka T, Sando Y, Suga T, Maeno Y, Nakagawa J, Hosono T, Sato M, Akiyama H, Kishi S: Stimulation of vascular endothelial growth factor gene transcription by all trans retinoic acid through spl and sp3 sites in human bronchioloalveolarcarcinoma cells. Am J Respir Cell Mol Biol 2002, 26(2):246-253.

16. Shi Q, Le X, Abbruzzese JL, Peng Z, Qian CN, Tang H, Xiong Q, Wang B, Li $X C$, Xie K: Constitutive Sp1 activity is essential for differential constitutive expression of vascular endothelial growth factor in human pancreatic adenocarcinoma 1. Cancer Res 2001, 61(10):4143-4154

17. Black AR, Black JD, Azizkhan-Clifford J: Sp1 and krüppel-like factor family of transcription factors in cell growth regulation and cancer. J Cell Physiol 2001, 188(2):143-160.

18. Li L, He S, Sun JM, Davie JR: Gene regulation by Sp1 and Sp3. Biochem Cell Biol 2004, 82(4):460-471.

19. Ryuto M, Ono M, Izumi H, Yoshida S, Weich HA, Kohno K, Kuwano M: Induction of vascular endothelial growth factor by tumor necrosis factor a in human glioma cells. J Biol Chem 1996, 271(45):28220.

20. Tanaka T, Kanai H, Sekiguchi K, Aihara Y, Yokoyama T, Arai M, Kanda T, Nagai $R$, Kurabayashi M: Induction of VEGF gene transcription by IL-1 [beta] is mediated through stress-activated MAP kinases and Sp1 sites in cardiac myocytes. J Mol Cell Cardiol 2000, 32(11):1955-1967.

21. Benckert C, Jonas S, Cramer T, von Marschall Z, Schäfer G, Peters M, Wagner K, Radke C, Wiedenmann B, Neuhaus P: Transforming growth factor $\beta 1$ stimulates vascular endothelial growth factor gene transcription in human cholangiocellular carcinoma cells. Cancer Res 2003, 63(5):1083.

22. Abdelrahim M, Smith R 3rd, Burghardt R, Safe S: Role of Sp proteins in regulation of vascular endothelial growth factor expression and proliferation of pancreatic cancer cells. Cancer Res 2004, 64(18):6740-6749.

23. Mabjeesh NJ, Willard MT, Frederickson CE, Zhong H, Simons JW: Androgens stimulate hypoxia-inducible factor 1 activation via autocrine loop of tyrosine kinase receptor/phosphatidylinositol 3/-kinase/protein kinase B in prostate cancer cells. Clin Cancer Res 2003, 9(7):2416.

24. Eisermann K, Bazarov A, Brett A, Knapp E, Piontkivska H, Fraizer G: Uncovering Androgen responsive regulatory networks in prostate cancer. In Proceedings of the Ohio Collaborative Conference on Bioinformatics. Washington, DC: IEEE Computer Society; 2009:99-103. doi:10.1109/ OCCBIO.2009.21. ISBN 978-0-7695-3685-9.

25. Lu S, Jenster G, Epner DE: Androgen induction of cyclin-dependent kinase inhibitor p21 gene: role of androgen receptor and transcription factor Sp1 complex. Mol Endocrinol 2000, 14(5):753-760.

26. Cleutjens KBJM, van der Korput HAGM, van Eekelen CCEM, van Rooij HCJ, Faber PW, Trapman J: An androgen response element in a far upstream enhancer region is essential for high, androgen-regulated activity of the prostate-specific antigen promoter. Mol Endocrinol 1997, 11(2):148.

27. Schäfer G, Cramer T, Suske G, Kemmner W, Wiedenmann B, Höcker M: Oxidative stress regulates vascular endothelial growth factor-a gene transcription through Sp1-and Sp3-dependent activation of two proximal GC-rich promoter elements. J Biol Chem 2003, 278(10):8190

28. Perez-Stable CM, Pozas A, Roos BA: A role for GATA transcription factors in the androgen regulation of the prostate-specific antigen gene enhancer. Mol Cell Endocrinol 2000, 167(1-2):43-53.

29. Chen PH, Tsao YP, Wang CC, Chen SL: Nuclear receptor interaction protein, a coactivator of androgen receptors (AR), is regulated by $A R$ and $\mathrm{Sp} 1$ to feed forward and activate its own gene expression through AR protein stability. Nucleic Acids Res 2008, 36(1):51-66.

30. Livak KJ, Schmittgen TD: Analysis of relative gene expression data using real-time quantitative PCR and the 2(-Delta Delta $C(T)$ ) Method. Methods 2001, 25(4):402-408

doi:10.1186/1476-4598-12-7

Cite this article as: Eisermann et al:: Androgen up-regulates vascular endothelial growth factor expression in prostate cancer cells via an Sp1 binding site. Molecular Cancer 2013 12:7. 